\title{
Aspirin inhibits colon cancer cell line migration through regulating epithelial-mesenchymal transition via Wnt signaling
}

\author{
SHENGHANG JIN and XIANGUO WU \\ Department of Clinical Laboratory, The Second Affiliated Hospital, \\ Zhejiang University School of Medicine, Hangzhou, Zhejiang 310009, P.R. China
}

Received January 17, 2018; Accepted January 31, 2019

DOI: $10.3892 /$ ol.2019.10089

\begin{abstract}
The mechanism responsible for the initiation of tumor metastasis and epithelial-mesenchymal transition (EMT) is not well understood. During EMT, epithelial cells lose their polarity and adhesion to surrounding cells and migrate, resulting in transition into mesenchymal cells. Canonical Wnt signaling has been implicated in controlling gene transcription and body axis pattern formation during development. However, canonical Wnt signaling has also been indicated to serve a role in carcinogenesis by regulating EMT. In the present study, it was demonstrated that the expression of several positive regulators of EMT and Wnt signaling was repressed by aspirin treatment in SW480 tumor cells, and that this reduction was due to alterations in the localization of zinc finger E-box binding homeobox 1 and Snail family transcriptional repressor 2. It was also demonstrated that aspirin may be an effective inhibitor of EMT, reducing the viability and migration ability of SW480 tumor cells, including cells induced by TGF- $\beta 1$.
\end{abstract}

\section{Introduction}

The source of mesenchymal cells in pathological processes, including tumor invasiveness and metastasis, is still not fully understood; however, increasing evidence suggests that epithelial-mesenchymal transition (EMT) is one of the important processes that lead to the formation of these mesenchymal cells (1). EMT is the process by which a polarized epithelial cell, which normally interacts with the basement membrane via its basal surface, undergoes multiple biochemical alterations that enable it to assume a mesenchymal cell phenotype,

Correspondence to: Dr Xianguo Wu, Department of Clinical Laboratory, The Second Affiliated Hospital, Zhejiang University School of Medicine, 88 Jiefang Road, Hangzhou, Zhejiang 310009, P.R. China

E-mail: xgw@zju.edu.cn

Key words: epithelial-mesenchymal transition,aspirin, Snail family transcriptional repressor 2, transnuclear, cell migration including enhanced migratory capacity, invasiveness and elevated resistance to apoptosis (2). Accumulating studies point to a critical series of EMT-like events during tumor progression and malignant transformation, providing the incipient cancer cell with invasive and metastatic properties (3-5). EMT may lead to the metastasis of cancer cells from the primary tumor into surrounding tissue and vasculature (6). Notably, preventing metastasis is the ultimate challenge in overcoming cancer (7).

The hallmark of EMT is the loss of epithelial surface markers, most notably epithelial (E)-cadherin and the acquisition of mesenchymal markers, including Vimentin, and neural $(\mathrm{N})$-cadherin. Downregulation of E-cadherin during EMT can be mediated by its transcriptional repression through the binding of EMT transcription factors including Snail, Snail family transcriptional repressor 2 (Slug) and twist family bHLH transcription factor 1 (Twist) to E-boxes present in the E-cadherin promoter (8). Slug is a Snail family transcription factor, which has been demonstrated to be involved in the control of apoptosis (9). Over expression of Slug has been identified in a number of different cancer types and is correlated with increases in tumor cell proliferation and lymph node metastasis (10).

Wnt signaling is an evolutionarily conserved pathway in animals involved with development but is also implicated in tumor formation. Wnt signaling serves a role in specifying cell fate, body axis patterning, proliferation and migration, which are necessary processes in the formation of vital tissues during embryonic development (11). Wnt mutations have been demonstrated to cause multiple diseases, including breast and prostate cancer, glioblastoma and diabetes. Typically, a Wnt protein binds to the domain of a member of the Frizzled family of receptors. These transmembrane G-protein coupled receptors send a signal to the Disheveled phosphoproteins located in the cytoplasm through direct interactions (12).

Dysregulationof canonical Wnt signaling processes can easily lead to cellular over-proliferation and tumor formation. Common dysregulations leading to tumor formation include mutations in components of the $\beta$-catenin destruction complex, overexpression of Wnt ligands, mutations in $\beta$-catenin and loss of other Wnt inhibitors and regulators (13). A previous study has demonstrated that repression of $\mathrm{Wnt} / \beta$-catenin signaling reduces EMT and suppresses metastasis of breast cancer to the lungs (14). 
In this study, two important inducers of EMT: Slug and zinc finger E-box binding homeobox 1 (ZEB1) were focused on. The two proteins bind to E-box consensus sequences on the E-cadherin promoter (15). These proteins facilitate EMT by directly repressing transcription of E-cadherin and other junctional proteins, including claudins and desmosomes (16). Notably, Wnt signaling induces Slug and the mesenchymal marker, Vimentin. Expression of Slug and ZEB1 are induced by the presence of transforming growth factor (TGF)- $\beta$ during embryogenesis to regulate the formation of different organs; however, certain types of metastatic cancer have been demonstrated to activate TGF- $\beta$ signaling (17).

Aspirin is one of the oldest known and most widely used medicines. It is known to reduce inflammation by irreversibly inhibiting cyclooxygenase (COX)-1 and COX-2 (18), inhibiting the formation of inflammatory prostaglandins (PGs) and eicosanoids by disrupting COX's formation of their precursors from arachidonic acid (17). Aspirin inhibits the activation of the Wnt signaling pathway by modulating Wnt/ $\mathrm{PGE}_{2}$ crosstalk and by promoting the degradation of $\beta$-catenin (19). Recently, there has been an increased focus on the role of aspirin as a chemopreventative drug. In the preset study it was demonstrated that in addition to its anti-inflammatory and cardioprotective characteristics, aspirin serves a role in inhibiting $\operatorname{EMT}(20,21)$.

\section{Materials and methods}

Cell line and reagents. The human colorectal adenocarcinoma SW480 cell line was obtained from the American Type Culture Collection (ATCC, Manassas, VA, USA). Cells were maintained in RPMI-1640 with 10\% fetal bovine serum (both from Thermo Fisher Scientific, Inc., Waltham, MA, USA). All associated antibodies [E-cadherin (cat. no. ab15148; 1:1,000)], $\mathrm{N}$-cadherin (cat. no. ab18203; 1:1,000), Vimentin (cat. no. ab8978; 1:3,000), Slug (cat. no. ab27568; 1:1,000), Snail (cat. no. ab53519; 1:1,000), Twist (cat. no. ab50581; 1:2,000), ZEB1 (cat. no. ab124512; 1:1,000), ZEB2 (cat. no. ab138222; 1:1,000) and GAPDH (cat. no. ab8245; 1:5,000) were purchased from Abcam (Cambridge, UK). Aspirin and TGF- $\beta 1$ were available from Sigma-Aldrich; Merck KGaA (Darmstadt, Germany).

Cell migration assay. Cell were plated at $1 \times 10^{5}$ cells/well in $200 \mu \mathrm{l}$ of culture medium in the upper chamber of Transwell permeable supports (Corning Inc., Corning, NY, USA; with an $8.0-\mu \mathrm{m}$ pore size, polycarbonate membrane, $6.5-\mathrm{mm}$ diameter and a 24-well plate format) to track the migration of SW480 cells. Migrated cells incubated in DMEM+10\% FBS on the lower surface of the membranes were fixed with $4 \%$ paraformaldehyde for $10 \mathrm{~min}$ at $37^{\circ} \mathrm{C}$ and stained with hexamethylpararosaniline chloride for $20 \mathrm{~min}$ at $37^{\circ} \mathrm{C}$. The number of cells was counted in three randomly selected fields of the wells under a light microscope.

Plasmid construction and cell transfection. The E-cadherin promoter region was cloned by polymerase chain reaction (PCR) and inserted into PGL-3 basic plasmid (cat. no. VT1554; YouBio, Hunan, China) which expressed the luciferase-reporting gene. The recombinant plasmid $(5 \mu \mathrm{g})$ was transferred into competent cells and positive clones were identified by PCR and restriction enzyme digestion prior tobeing sequenced. Followingthe amplification of recombinant plasmid using a QIAGEN Plasmid Maxi kit (12162; Qiagen, Inc., Valencia, CA, USA), the E-cadherin luciferase report plasmid was used for cell transfection. For the ZEB-RFP and Slug-RFP constructs, cDNA fragments of ZEB and Slug were amplified by PCR and inserted into PCDNA3.1-RFP vector (cat. no. VPY0003; Yrgene, Changsha, China). Briefly, a large number of ZEB-RFP and Slug-RFP plasmids were obtained according to the above step. The siRNAs targeting ZEBl gene were designed and synthesized by the Shanghai GenePharma Co., Ltd., (Shanghai, China). Small interfering (si)RNA sequences for three sites of the ZEBI gene are listed in Table I. Transfections (10 $\mu$ mol siRNA) were performed using the Lipofectamine ${ }^{\circledR} 2000$ kit (Thermo Fisher Scientific, Inc.) according to the manufacturer's protocol.

Cell proliferation assay. SW480 cells were seeded in 96-well plates. Cell proliferation was assessed using a Cell counting Kit-8 (CCK-8; cat.no. 1166/1; R\&D Systems,Inc., Minneapolis, MN, USA) according to the manufacturer's protocol. The number of cells was measured using a microplate reader at a wavelength of $450 \mathrm{~nm}$.

Reverse transcription-quantitative PCR (RT-qPCR). Total RNA was extracted from the cells using TRIzol reagent (Invitrogen; Thermo Fisher Scientific, Inc.) according to the manufacturer's protocol. cDNA was synthesized by adding purified RNA and oligo(dT) primers to SuperScript II at $4^{\circ} \mathrm{C}$ (Invitrogen; Thermo Fisher Scientific, Inc.). QPCR was performed using SYBR-Green (Applied Biosystems; Thermo Fisher Scientific, Inc.) according to the manufacturer's protocol (primers are listed in Table II). The reactions were performed as follows: $95^{\circ} \mathrm{C}$ for $15 \mathrm{~min}, 95^{\circ} \mathrm{C}$ for $10 \mathrm{sec}, 60^{\circ} \mathrm{C}$ for $32 \mathrm{sec}$, $72^{\circ} \mathrm{C}$ for $32 \mathrm{sec}$ and $85^{\circ} \mathrm{C}$ for $6 \mathrm{sec}$ for 40 cycles; the melting curve analysis ranging from 60 to $95^{\circ} \mathrm{C}$, gradually increased at a speed of $0.5 \mathrm{kC}$ every $10 \mathrm{sec}$. The relative expressions levels were normalized to GAPDH and expressed using the $2^{-\Delta \Delta C t}$ method (22).

Western blotting. Cells were lysed in ice-cold radioimmunoprecipitation buffer containing a protease inhibitor cocktail (RocheDiagnostics, Basel, Switzerland). The concentration of protein was measured using a Bio-Rad protein assay kit (Bio-Rad Laboratories, Inc., Hercules, CA, USA) according to the manufacturer's protocol. Proteins $(60 \mu \mathrm{g})$ were separated by $8 \%$ SDS-PAGE and were transferred onto nitrocellulose membranes. The membranes were blocked in PBS containing $5 \%$ skimmed milk for $1 \mathrm{~h}$ at $37^{\circ} \mathrm{C}$ and incubated overnight at $4^{\circ} \mathrm{C}$ with the primary antibodies against the protein of interest. Goat anti-mouse immunoglobulin (Ig)G H\&L horseradish peroxidase (HRP; cat. no. ab6789; 1:5,000; Abcam) and goat anti-rabbit IgG H\&L (HRP; cat. no. ab6721; 1:5,000; Abcam) were then added to each membrane and incubated for $2 \mathrm{~h}$ at $37^{\circ} \mathrm{C}$. The presence of target proteins was detected using enhanced chemiluminescence system (cat. no. K820-50; BioVision, Inc., Milpitas, CA, USA). Each protein band was analyzed by densitometry using Image-Pro Plus 6.0 software (National Institutes of Health, Bethesda, MD, USA) and normalized to expression of the internal control GAPDH. 
Table I. siRNA sequences for zinc finger E-box binding homeobox1 gene silencing.

\begin{tabular}{llc}
\hline Site & \multicolumn{1}{c}{ Sense } & Antisense \\
\hline siRNA-313 & CCAUGAUCCUAAUGUUGAAGA & UUCAACAUUAGGAUCAUGGUU \\
siRNA-1227 & GGCAAGUGUUGGAGAAUAAUC & UUAUUCUCCAACACUUGCCUU \\
siRNA-1963 & GGACAGCACAGUAAAUCUACA & UAGAUUUGCUGUGCUGUCCUG
\end{tabular}

Si, small interfering.

Table II. Primers for reverse transcription-quantitative polymerase chain reaction.

\begin{tabular}{lll}
\hline Gene & \multicolumn{1}{c}{ Forward sequence } & \multicolumn{1}{c}{ Reverse sequence } \\
\hline E-cadherin & 5'-CTGAGAACGAGGCTAACG-3' & 5'-TTCACATCCAGCACATCC-3' \\
N-cadherin & 5'-ATCCTACTGGACGGTTCG-3' & 5'-TTGGCTAATGGCACTTGA-3' \\
Vimentin & 5'-CCAGGCAAAGCAGGAGTC-3' & 5'-CGAAGGTGACGAGCCATT-3' \\
Slug & 5'-AGGAATCTGGCTGCTGTG-3' & 5'-ATGCCTTTGGACTTTATTTGTC-3' \\
Snail & 5'-ACAAGCACCAAGAGTCCG-3' & 5'-CCCTCCCTCCACAGAAAT-3' \\
Twist & 5'-CGGGAGTCCGCAGTCTTA-3' & 5'-CACGCCCTGTTTCTTTGA-3' \\
ZEB1 & 5'-AAGTGGCGGTAGATGGTA-3' & 5'-TGTTGTATGGGTGAAGCA-3' \\
ZEB2 & 5'-TATTCCTGGGCTACGACC-3' & 5'-TCTCCCTGAAATCCTTGT-3'
\end{tabular}

E, epithelial; N, neural; ZEB, zinc finger E-box binding homeobox; Snail, snail family transcriptional repressor 1; slug, snail family transcriptional repressor 2; twist, twist family bHLH transcription factor 1.

Immunofluorescence. SW480 cells transfected with ZEB-RFP and Slug-RFP plasmids were seeded on cell culture slides in 12 -well plates at $5 \times 10^{5}$ cells/well and grown until $40 \%$ confluence. Following treatment with TGF- $\beta 1$ and TGF- $\beta 1+$ aspirin for $24 \mathrm{~h}$, cells were fixed with $4 \%$ paraformaldehyde for $30 \mathrm{~min}$ at $37^{\circ} \mathrm{C}$ and washed three times with PBS (5 min each time). Next, cells were incubated with $1 \%$ Triton $\mathrm{X}-100$ for $10 \mathrm{~min}$. After washing with $\mathrm{PBS}, 3 \% \mathrm{H}_{2} \mathrm{O}_{2}$ was added in order to neutralize endogenous peroxidase. The glass slide containing cells was blocked using 5\% bovine serum albumin (cat. no. A1933, Sigma-Aldrich; Merck KGaA) for $30 \mathrm{~min}$ and the samples were incubated with primary antibodies against ZEB-1 (1:100) and Slug $(1: 100)$ overnight at $4^{\circ} \mathrm{C}$. The next day, secondary antibody goat-anti-rabbit-596 $\mathrm{nm}(1: 400)$ was added to the cells and incubated for $2 \mathrm{~h}$ at room temperature. DAPI staining (10 $\mathrm{min}$ ) was used to label cell nuclei at $37^{\circ} \mathrm{C}$. Subsequently, slides were mounted using mounting medium and signals were observed using a fluorescence microscope.

Luciferase reporter assay. SW480 colon cancer cells were seeded at a density of $1 \times 10^{5}$ cells/well in 48 -well plates. The firefly luciferase reporter gene assay kit (Shanghai Kangnian Biotechnology Co., Ltd., Shangai, China) was performed in uninduced and TGF- $\beta 1$-induced SW480 cells and in aspirin-treated and untreated (10 ng/ml) SW480 cells. Samples $(10 \mu \mathrm{l})$ were assayed for Gaussia luciferase activity using a microplate luminometer in the Renilla luciferase assay buffer. A simple Gaussia luciferase assay buffer (PBS containing $1.43 \mu \mathrm{M}$ coelenterazine) was also tested and yielded similar results. Luciferase activity was measured with a luminometer
(Promega Corporation, Madison, WI, USA) according to the manufacturer's protocol.

Statistical analysis. The data were analyzed by one-way analysis of variance with a Tukey's post-hoc test using GraphPad Prism 5 (GraphPad Software Inc., La Jolla, CA, USA). The data were expressed as the mean \pm standard deviation. $\mathrm{P}<0.05$ was considered to indicate a statistically significant difference. Each experiment was repeated three times.

\section{Results}

Aspirin inhibits EMT due to its anti-inflammatory and Wnt inhibitor effects. This hypothesis was tested by treating SW480 cells and measuring their viability and migration ability via the Transwell assay. A line of Dukes' type B, human colorectal adenocarcinoma SW480 cells was obtained from the ATCC. These cells have a mutation in codon 12 of the ras proto-oncogene and express $C$-myc, K-ras, $H$-ras, $N$-ras, Myb, Sis and Fos oncogenes. This line also has a $\mathrm{G}$ to A mutation in p53 and forms tumors at $100 \%$ frequency following 21 days in mice.

Aspirin inhibits the viability and migration ability of colon cancer SW480 cells. SW480 cells were cultured in the presence of $0.5-10 \mathrm{mM}$ aspirin for 2 days and the results were quantified using a CCK-8 kit. A decreased level of viable cells was observed as the concentration of aspirin was increased. To determine the effect of aspirin on SW480 cell migration, SW480 cells were grown on a membrane and treated with 0.5-10 $\mathrm{mM}$ aspirin for 2 days. A CCK- 8 was used to quantify 

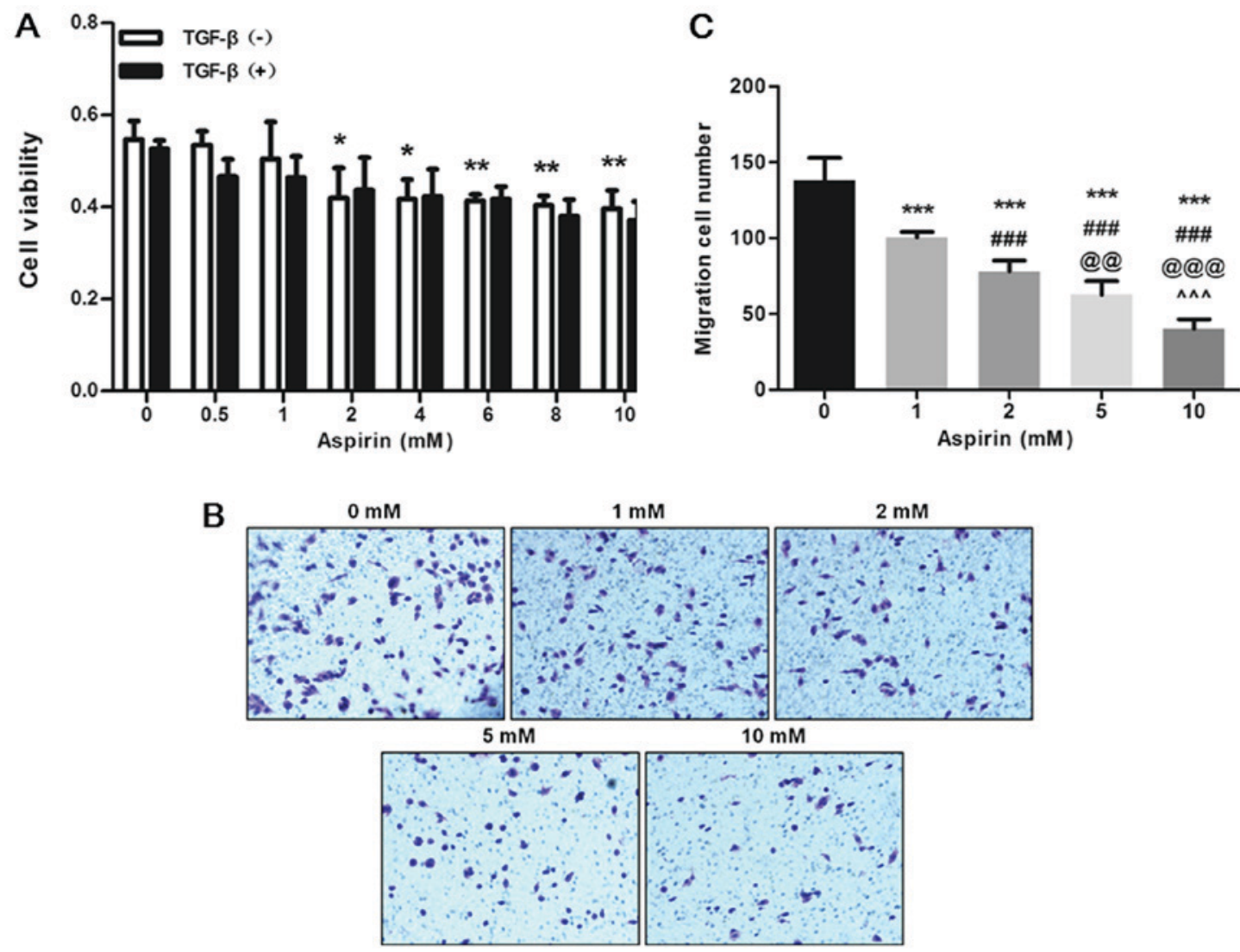

Figure 1. Aspirin reduces the proliferation and migration ability of SW480 cells. (A) The effect of aspirin on cell viability was determined. TGF- $\beta$-induced and uninduced cells were treated with $0.5-10 \mathrm{mM}$ aspirin for 2 days and cell proliferation was determined using a Cell Counting Kit- 8 kit. "P<0.05 TGF- $\beta$ (-) and TGF- $\beta(+)$ group. (B) The inhibitory effect of aspirin on SW480 cell migration ability was assessed using a Transwell assay. Cells were stained with hexamethylpararosaniline chloride and observed under a light microscope. Magnification, x100. (C) Relative quantitation of the number of migrated cells observed in panel B was performed. ${ }^{*} \mathrm{P}<0.05,{ }^{* *} \mathrm{P}<0.01$ and ${ }^{* * * *} \mathrm{P}<0.001$ vs. non-treated group; ${ }^{\# \# \#} \mathrm{P}<0.001$ vs. $1 \mathrm{mM}$ aspirin; ${ }^{\circledR @} \mathrm{P}<0.01$ and ${ }^{\circledR @ @ ~} \mathrm{P}<0.001$ vs. $2 \mathrm{mM}$ aspirin; ${ }^{\wedge \wedge} \mathrm{P}<0.001$ vs. $5 \mathrm{mM}$ aspirin. TGF, transforming growth factor.

the number of cells that had migrated from the membrane. The results indicated that aspirin reduced the migratory ability of SW480 cells (Fig. 1).

Aspirin reduces TGF- $\beta$-induced ETM in colon cancer $S W 480$ cells. In order to determine the effects of aspirin on the SW480 cancer cells that had been induced by TGF- $\beta 1$, SW480 cells were cultured and treated for $24 \mathrm{~h}$ with $5 \mathrm{ng} / \mathrm{mlTGF}-\beta 1$ in order to promote their differentiation into a mesenchymal cell type. Cells were subsequently treated with $10 \mathrm{mM}$ aspirin. The transdifferentiated cells were cultured, their viability was determined and another Transwell assay was performed to assess the effect of aspirin on the ability of cells to migrate. It was observed that the application of aspirin reduced the migration ability and viability of TGF- $\beta 1$-induced cells (Fig. 2A). mRNA was extracted from treated cells and RT-qPCR wasused to determine the relative expression of several EMT-associated genes: E-cadherin, $N$-cadherin, Vimentin, Slug, Snail, Twist, ZEB1 and ZEB2. The application of aspirin increased the relative expression of epithelial cell marker $E$-cadherin but decreased the relative expression levels of mesenchymal marker $N$-cadherin. As indicated in Fig. 2B, $N$-cadherin, Vimentin, Slug, Snail, Twist, ZEB1 and ZEB2, which are all inducers of Wnt signaling and positive regulators of EMT, were decreased in aspirin-treated samples. In order to determine if the changes to relative gene expression levels observed caused a change to the protein levels, a western blot was performed on TGF- $\beta 1$-exposed samples with or without aspirin treatment. Notably, similar effects with regards to the protein expression levels of the corresponding assayed genes were observed (Fig. 2C).

To further confirm the effects of aspirin on TGF- $\beta 1$-induced SW480 cells, a luciferase-based reporter system was used. A construct was generated by fusing a luciferase reporter gene with the promoter of E-cadherin. SW480 cells were transformed with this reporter construct and treated as above. Cells treated with aspirin exhibited significantly increased luciferase activity in the TGF- $\beta 1$-induced and uninduced cells when compared with untreated cells ( $\mathrm{P}<0.001$; Fig. 3).

Aspirin inhibits the transnuclear behavior of Slug and ZEB1 in colon cancer SW480 cells. It was hypothesized that the increase in E-cadherin: Luciferase reporter activity in aspirin-treated cells was caused by a change to the localization of a negative regulator of $E$-cadherin expression. A previous study has revealed that ZEB1 and ZEB2 can directly bind the E-cadherin promoter to inhibit E-cadherin expression (23). Therefore, the nuclear protein fraction from aspirin-treated and untreated TGF- $\beta 1$-induced cells was isolated. A western blot was performed to determine the expression levels of ZEB1, ZEB2 and Slug in the nuclear and cytoplasmic fractions. Results indicated that the expression levels of ZEB1, 
A

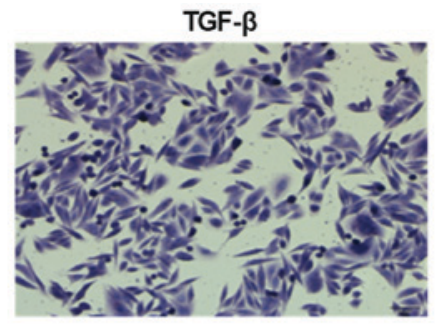

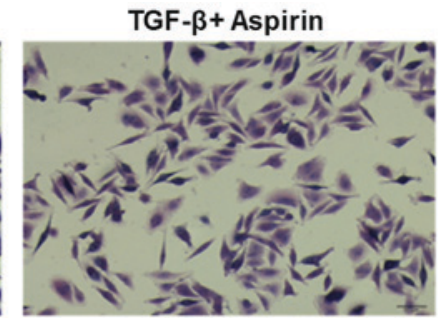

B

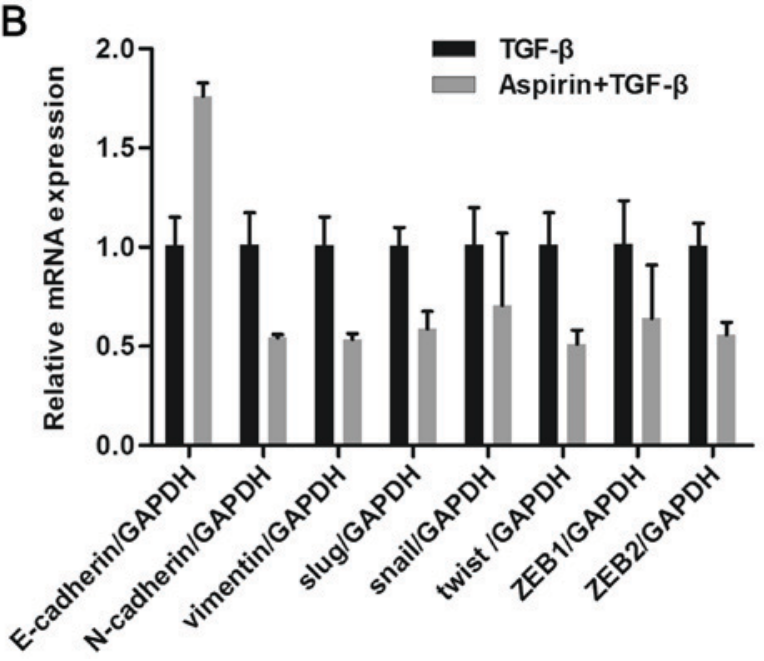

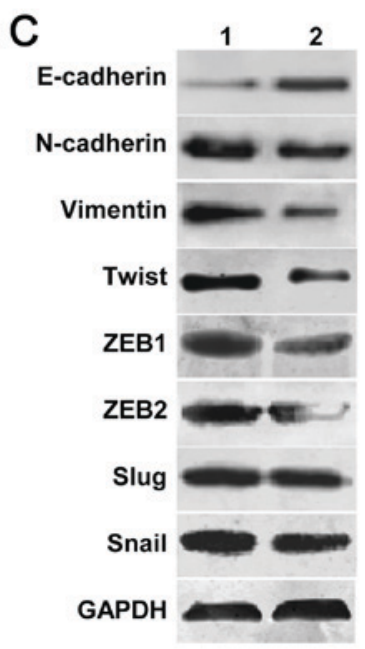

Figure 2. Aspirin reduces TGF- $\beta$-induced epithelial-mesenchymal transition. (A) Inhibition of aspirin on EMT in SW480 was determined. Cellular migration was measured via a Transwell assay. TGF- $\beta(5 \mathrm{ng} / \mathrm{ml}$ for $24 \mathrm{~h})$ was added to cells to induce epithelial mesenchymal transformation. Magnification, $x 100$. (B) Transcriptional levels of EMT-associated genes were detected by reverse transcription-quantitative polymerase chain reaction. (C) Protein expression levels of EMT-associated genes were detected by western blotting. Notably, aspirin influenced the levels of E-cadherin, $\mathrm{N}$-cadherin and vimentin in the process of EMT, resulting in the alterations of cell morphology. EMT, epithelial mesenchymal transition; TGF, transforming growth factor; e, epithelial; N, neural; ZEB, zinc finger E-box binding homeobox; Snail, snail family transcriptional repressor 1; slug, snail family transcriptional repressor 2; twist, twist family bHLH transcription factor 1 .

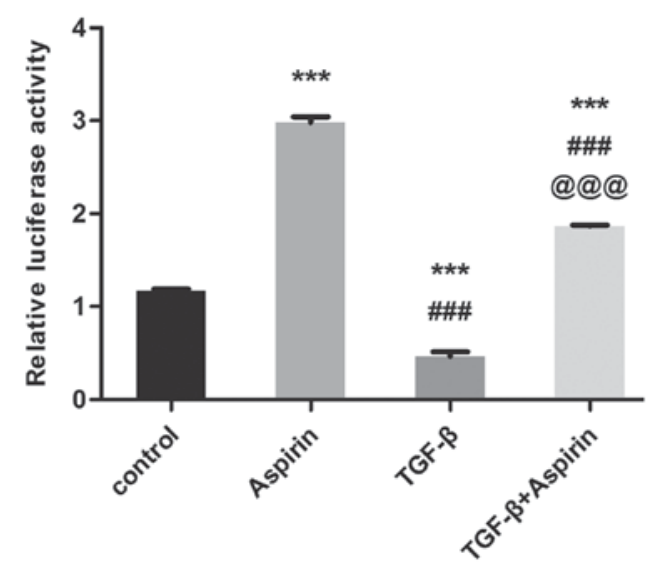

Figure 3. Confirmation of the effects of aspirin on TGF- $\beta 1$-induced EMT using the luciferase reporter assay. Relative luciferase activity was measured in cells treated with TGF- $\beta 1$, aspirin and TGF- $\beta 1+$ aspirin. Non-aspirin-treated and non-TGF- $\beta$-treated cells were used as negative controls. ${ }^{* * *} \mathrm{P}<0.001 \mathrm{vs.} \mathrm{the}$ control; ${ }^{\# \# ~} \mathrm{P}<0.001$ vs. the aspirin only; ${ }^{\circledR @ @ ~} \mathrm{P}<0.001$ vs. the TGF- $\beta$ only group. TGF, transforming growth factor.

ZEB2 and Slug were increased in the cytoplasm and decreased in the nucleus of aspirin-treated cells (Fig. 4A). To confirm the localization of ZEB1 and Slug, an immunofluorometric assay was used. A construct was generated using ZEB1 and Slug fusion with RFP and the SW480 cells were transformed with the constructs. DAPI was used to stain the nuclei of the cells.
Following aspirin treatment and immunostaining, ZEB1-RFP and Slug-RFP signals were localized in the cytoplasm in aspirin-treated cells, whereas in untreated cells ZEB1-RFP and Slug-RFP signals were localized in the nucleus (Fig. 4B). These data led to the conclusion that aspirin inhibits that transport of Slug and ZEB1 to the nucleus in TGF- $\beta 1$-induced, aspirin-treated SW480 cells.

Aspirin inhibits EMT in ZEB1 knockdown of SW480 cells. After observing that aspirin could impact the nuclear transport of Slug and ZEB1, it was hypothesized that a similar inhibitory effect on EMT could be achieved by knocking down ZEB1. In order to achieve this, different siRNA constructs were generated and three were selected that could successfully knock down ZEB1: siRNA-313, siRNA-1227 and siRNA-1963. siRNA-NC, a non-complementary siRNA to ZEBl, was used as a control. The relative amount of $Z E B 1$ mRNA was quantified using RT-qPCR following isolation of the total mRNA from transformed SW480 cells (Fig. 5A). Successful knockdown of the expression level of ZEB1 mRNA was observed and a western blot was then conducted to confirm that this knockdown could successfully reduce the expression of ZEB1 protein (Fig. 5B). The western blot confirmed that the knockdown siRNA constructs were working effectively. siRNA ZEBI- and siRNA NC-transformed cells were then induced with TGF- $\beta 1$ as indicated above and treated with aspirin (Fig. 5C). A western blot was performed to assess the expression level of 


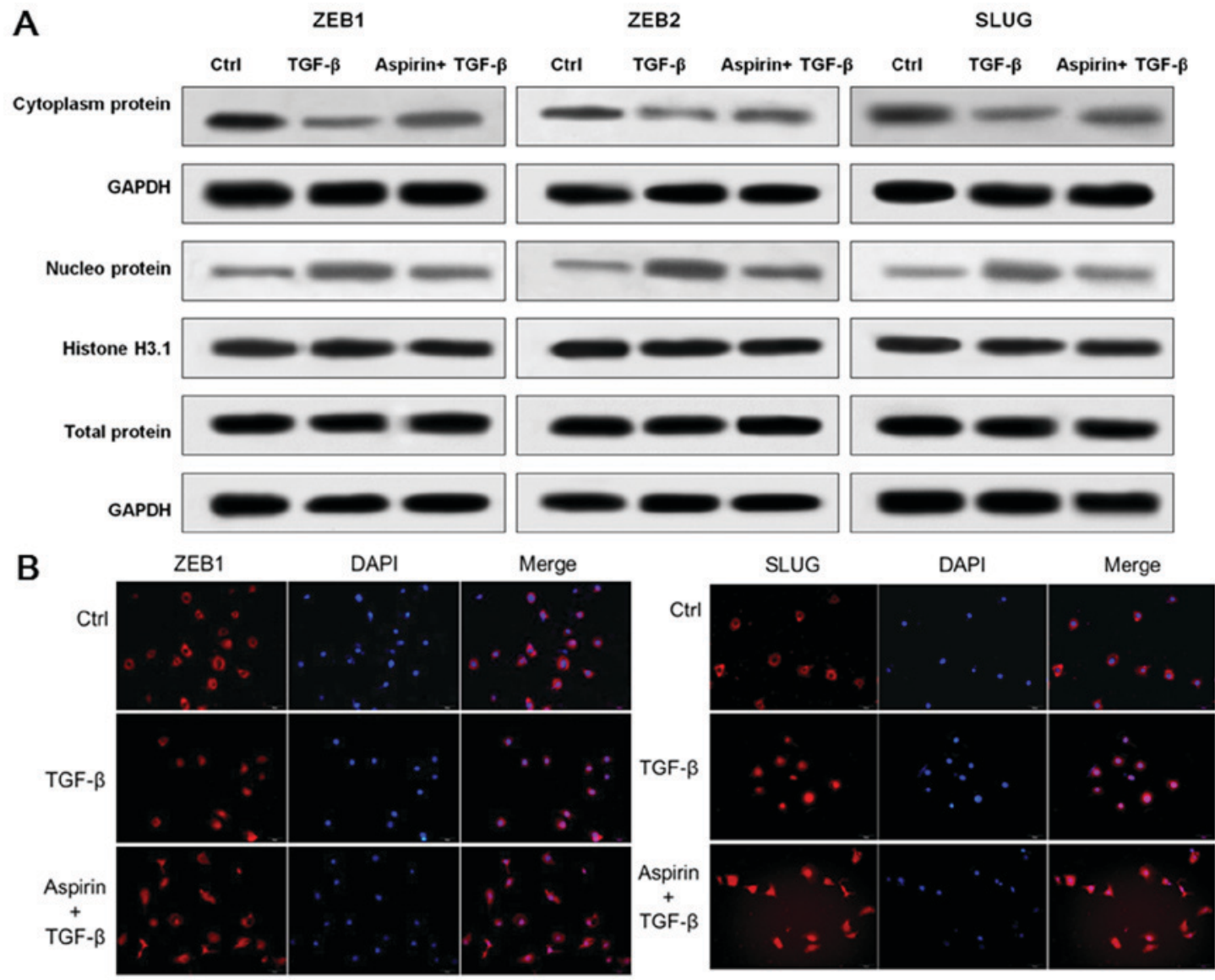

Figure 4. Aspirin inhibits the transnuclear behavior of Slug and ZEB1 in SW480 colon cancer cells. (A) Detection of Slug, ZEB1 and ZEB2 protein expression levels in the nucleus and cytoplasm by western blotting. (B) Slug and ZEB1 localization was indicated using immunofluorometric assay; magnification, 200x. ZEB, zinc finger E-box binding homeobox; slug, snail family transcriptional repressor 2; Ctrl, control; DAPI, 4',6-diamidino-2-phenylindole.

E-cadherin present in these cells. It was observed that siRNA ZEB1-transformed cells exhibited an increased expression level of E-cadherin and aspirin treatment further increased the level of E-cadherin present, augmenting the effect of siRNA $Z E B 1$. Furthermore, the transformed cells were cultured and a Transwell assay was performed as above followinginduction with TGF- $\beta 1$ and aspirin treatment. Cells that received no treatment with aspirin and siRNA NC-transformed cells were used as controls. Notably, a significant reduction in the migratory ability of SW480 cells that had been treated with siRNA ZEB1 was indicated compared with the NC group $(\mathrm{P}<0.01$; Fig. 5D and E). Furthermore, this reduction was further reduced by aspirin treatment, reflecting the increase in E-cadherin that was observed earlier. As presentedin Fig. 5E, relative quantitation of the number of migrated cells demonstratedthe same tendency with transwell assay (Fig. 5E).

\section{Discussion}

The findings presented above convincingly suggested that aspirin may be an effective drug for inhibiting the migration of neoplastic cells. The results demonstrated that aspirin caused SW480 cells to retain their epithelial surface markers but reduced the level of mesenchymal markers by limiting the nuclear transport of Slug and ZEB1. The results also suggested that the absence of ZEB1 may lead to transcriptional alterations, affecting Wnt signaling and further limiting EMT.
Previous studies have demonstrated that aspirin treatment causes a reduction in the level of $\beta$-catenin available for Wnt signaling and that this reduction is due to increased ubiquitination and increased phosphorylation sites on $\beta$-catenin, marking it for degradation $(24,25)$. In addition, a previous study indicated that aspirin can inactivate the PP2A complex and is involved in multiple levels of Wnt signaling via autophosphorylation (25). Given these previous findings, it is not unexpected that aspirin caused the reduction in EMT that was observed.

A total of three possible mechanisms were proposed to be responsible for the effects of aspirin treatment observed in the present study. Firstly, it was proposed that aspirin affects the nuclear transport of Slug. Previous studies have demonstrated that aspirin can downregulate the expression of Slug in aggressive $K$-ras-expressing cancer cells $(26,27)$. A previous study has also implicated aberrant nuclear transport of Snail family member transcription factors in tumor formation (28). Taken together, these data create a convincing foundation for a mechanism by which EMT is suppressed in aspirin-treated tumor cells. Secondly, aspirin may repress Wnt signaling. As mentioned above, aspirin can negatively regulate a number of proteins involved in Wnt signaling. Therefore, it is not surprising that a reduction of $\beta$-catenin caused by aspirin can reduce EMT. Thirdly, aspirin may alter ZEB1 acetylation, possibly affecting Slug acetylation. In H358 non-small-cell lung cancer cells, reduced acetylation was identified in histones 
A

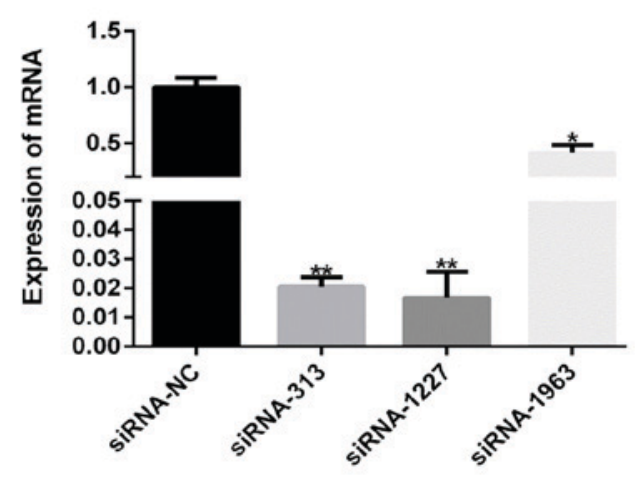

B

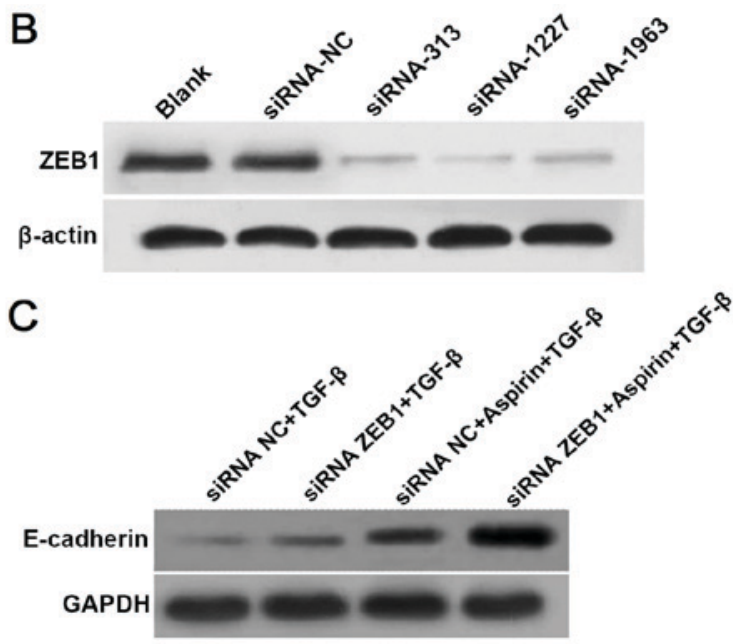

E

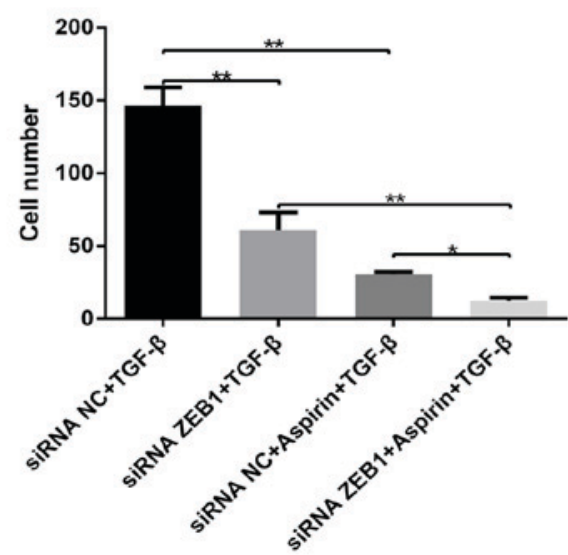

Figure 5. Aspirin inhibits epithelial mesenchymal transitionin ZEB1 knockdown of SW480 cells. Identification of ZEB1 gene silencing efficiency was indicated by (A) reverse transcription-quantitative polymerase and (B) western blotting. siRNA-31, siRNA-1227 and siRNA-1963 were selected to interfere with ZEBI expression. (C) Effects of ZEB1 gene silencing on E-cadherin expression level after aspirin treatment in TGF- $\beta$ 1-pretreated cells. (D) Cells were stained with hexamethylpararosaniline chloride and observed under a light microscope; magnification, 50x. (E) Relative quantitation of the number of positively-stained cells in panel $\mathrm{D}$ is indicated. ${ }^{*} \mathrm{P}<0.05$ and ${ }^{* *} \mathrm{P}<0.01$ vs. the siRNA-NC. Si, small interfering; NC, negative control; TGF, transforming growth factor; ZEB, zinc finger E-box binding homeobox; E, epithelial.

surrounding ZEB1 binding sites (29). Epigenetic alterations like these can increase the availability of ZEB1 binding sites in the promoters of endothelial marker genes. Previous research has also demonstrated that the deacetylase SIRT2 can rapidly destabilize Slug in basal-like breast cancer cells (30). These findings suggest aspirin may serve a role in affecting the two acetylation changes, inhibiting EMT.

The novel research in the present study highlights the positive effects aspirin has on inhibiting EMT. Taken together, these data suggest that aspirin can inhibit tumor metastasis by inhibiting tumor cells from acquiring the ability to invade surrounding tissue and circulate to other tissues. The data from the present study reflect results of previous findings, which have demonstrated that aspirin can increase the survival rate and reduce the occurrence of colorectal cancer in individuals that take aspirin on a regular basis (31). Although the present research provides insights into the effects of aspirin treatment on tumor cells, more remains to be discovered regarding the mechanism of action of aspirin. Recent studies suggest that aspirin has beneficial effects on preventing colorectal cancer; however, aspirin seems to have no effect on lowering the number of circulating tumor cells identified in metastatic breast cancer patients (32-34). In addition, much remains to be developed in the medical field before screening for EMT markers becomes commonplace. The advent of innovative EMT screening strategies in the clinical setting makes for an exciting outlook for future research.

\section{Acknowledgements}

Not applicable.

\section{Funding}

The present study was supported by the Natural Science Foundation of Zhejiang Province (grant no. LY15H160037) and the Education Bureau of Zhejiang Province (grant no. Y201330050).

\section{Availability of data and materials}

The datasets used and/or analyzed during the current study are available from the corresponding author on reasonable request. 


\section{Authors' contributions}

SJ performed all the experiments, drafting and revised the manuscript. XW participated in the development of the research concept and experimental design, and proposed revision suggestions. All the authors have read and approved the final manuscript.

\section{Ethics approval and consent to participate}

Not applicable.

\section{Patient consent for publication}

Not applicable.

\section{Competing interests}

The authors declare that they have no conflicts of interest.

\section{References}

1. Kalluri R and Weinberg RA: The basics of epithelial-mesenchymal transition. J Clin Invest 119: 1420-1428, 2009.

2. Kalluri R and Neilson EG: Epithelial-mesenchymal transition and its implications for fibrosis. J Clin Invest 112: 1776-1784, 2003.

3. Larue L and Bellacosa A: Epithelial-mesenchymal transition in development and cancer: Role of phosphatidylinositol 3' kinase/AKT pathways. Oncogene 24: 7443-7454, 2005.

4. Li J, Yang R, Dong Y, Chen M, Wang Y and Wang G: Knockdown of FOXO3a induces epithelial-mesenchymal transition and promotes metastasis of pancreatic ductal adenocarcinoma by activation of the $\beta$-catenin/TCF4 pathway through SPRY2. J Exp Clin Cancer Res 38: 38, 2019.

5. Hou X, Yang L, Jiang X, Liu Z, Li X, Xie S, Li G and Liu J: Role of microRNA-141-3p in the progression and metastasis of hepatocellular carcinoma cell. Int J Biol Macromol 128: 331-339, 2019.

6. Yamaguchi H, Wyckoff $\mathrm{J}$ and Condeelis $\mathrm{J}$ : Cell migration in tumors. CurrOpin Cell Biol 17: 559-564, 2005.

7. Wan L, Pantel K and Kang Y: Tumor metastasis: Moving new biological insights into the clinic. Nat Med 19: 1450-1464, 2013.

8. Serrano-Gomez SJ, Maziveyi M and Alahari SK: Regulation of epithelial-mesenchymal transition through epigenetic and post-translational modifications. Mol Cancer 15: 18, 2016.

9. Wang Y, Yue B, Yu X, Wang Z and Wang M: SLUG is activated by nuclear factor kappa B and confers human alveolar epithelial A549 cells resistance to tumor necrosis factor-alpha-induced apoptosis. World J Surg Oncol 11: 12, 2013.

10. Uchikado $Y$, Natsugoe $S$, Okumura $H$, Setoyama $T$, Matsumoto M, Ishigami S and Aikou T: Slug expression in the E-cadherin preserved tumors is related to prognosis in patients with esophageal squamous cell carcinoma. Clin Cancer Res 11: 1174-1180, 2005

11. Nusse R: Wnt signaling in disease and in development. Cell Res 15: 28-32, 2005

12. Rao TP and Kühl M: An updated overview on Wnt signaling pathways: A prelude for more. Circ Res 106: 1798-1806, 2010.

13. Micalizzi DS, Farabaugh SM and Ford HL: Epithelialmesenchymaltransition in cancer: Parallels between normal development and tumor progression. J Mammary Gland Biol Neoplasia 15: 117-134, 2010.

14. Jang GB, Kim JY, Cho SD, Park KS, Jung JY, Lee HY, Hong IS and Nam JS: Blockade of Wnt/ $\beta$-catenin signaling suppresses breast cancer metastasis by inhibiting CSC-like phenotype. Sci Rep 5: 12465, 2015.

15. Peinado H, Olmeda D and Cano A: Snail, Zeb and bHLH factors in tumour progression: An alliance against the epithelial phenotype? Nat Rev Cancer 7: 415-428, 2007.

16. Boyer B, Tucker GC, Vallés AM, Franke WW and Thiery JP: Rearrangements of desmosomal and cytoskeletal proteins during the transition from epithelial to fibroblastoid organization in cultured rat bladder carcinoma cells. J Cell Biol 109: 1495-1509, 1989.
17. Kang Y, He W, Tulley S, Gupta GP, Serganova I, Chen CR, Manova-Todorova K, Blasberg R, Gerald WL and Massagué J: Breast cancer bone metastasis mediated by the smad tumor suppressor pathway. Proc Natl Acad Sci USA 102: 13909-13914, 2005.

18. Loll PJ, Picot D and Garavito RM: The structural basis of aspirin activity inferred from the crystal structure of inactivated prostaglandin H2 synthase. Nat Struct Biol 2: 637-643, 1995.

19. Funk CD, Funk LB, Kennedy ME, Pong AS and Fitzgerald GA: Human platelet/erythroleukemia cell prostaglandin $\mathrm{G} / \mathrm{H}$ synthase: CDNA cloning, expression, and gene chromosomal assignment. FASEB J 5: 2304-2312, 1991.

20. Ying J, Zhou HY, Liu P, You Q, Kuang F, Shen YN and Hu ZQ: Aspirin inhibited the metastasis of colon cancer cells by inhibiting the expression of toll-like receptor 4. Cell Biosci 8: 1, 2018.

21. Liu Y, Yuan X, Li W, Cao Q and Shu Y: Aspirin-triggered resolvin D1 inhibits TGF- $\beta 1$-induced EMT through the inhibition of the mTOR pathway by reducing the expression of PKM2 and is closely linked to oxidative stress. Int J Mol Med 38: 1235-1242, 2016

22. Livak KJ and Schmittgen TD: Analysis of relative gene expression data using real-time quantitative PCR and the 2(-Delta Delta C(T)) method. Methods 25: 402-408, 2001.

23. Meyer-Schaller N, Heck C, Tiede S, Yilmaz M and Christofori G: Foxf2 plays a dual role during transforming growth factor beta-induced epithelial to mesenchymal transition by promoting apoptosis yet enabling cell junction dissolution and migration. Breast Cancer Res 20: 118, 2018.

24. Dihlmann S, Klein S and Doeberitz Mv Mv: Reduction of beta-catenin/T-Cell transcription factor signaling by aspirin and indomethacin is caused by an increased stabilization of phosphorylated beta-catenin. Mol Cancer Ther 2: 509-516, 2003.

25. Bos CL, Kodach LL, van den Brink GR, Diks SH, van Santen MM, Richel DJ, Peppelenbosch MP and Hardwick J: Effect of aspirin on the Wnt/beta-catenin pathway is mediated via protein phosphatase 2A. Oncogene 25: 6447-6456, 2006.

26. Khan P, Manna A, Saha S, Mohanty S, Mukherjee S, Mazumdar M, Guha D and Das T: Aspirin inhibits epithelial-to-mesenchymal transition and migration of oncogenic K-ras-expressing non-small cell lung carcinoma cells by down-regulating E-cadherin repressor slug. BMC Cancer 16: 39, 2016.

27. Petrera M, Paleari L, Clavarezza M, Puntoni M, Caviglia S, Briata IM, Oppezzi M, Mislej EM, Stabuc B, Gnant M, et al: The ASAMET trial: A randomized, phase II, double-blind, placebo-controlled, multicenter, $2 \times 2$ factorial biomarker study of tertiary prevention with low-dose aspirin and metformin in stage I-III colorectal cancer patients. BMC Cancer 18: 1210, 2018.

28. Muqbil I, Wu J, Aboukameel A, Mohammad RM and Azmi AS: Snail nuclear transport: The gateways regulating epithelialto-mesenchymaltransition? Semin Cancer Biol 27: 39-45, 2014.

29. Roche J, Nasarre P, Gemmill R, Baldys A, Pontis J, Korch C, Guilhot J, Ait-Si-Ali S and Drabkin H: Global decrease of histone H3K27 acetylation in ZEB1-induced epithelial to mesenchymal transition in lung cancer cells. Cancers (Basel) 5: 334-356, 2013.

30. Zhou W, Ni TK, Wronski A, Glass B, Skibinski A, Beck A and Kuperwasser C: The SIRT2 deacetylase stabilizes Slug to control malignancy of basal-like breast cancer. Cell Rep 17: 1302-1317, 2016.

31. Flossmann E and Rothwell PM; British Doctors Aspirin Trial and the UK-TIA Aspirin Trial: Effect of aspirin on long-term risk of colorectal cancer: Consistent evidence from randomised and observational studies. Lancet 369: 1603-1613, 2017.

32. Ni C and Yang L: Aspirin to inhibit the formation and EMT of circulating tumor cells in patients with metastatic colorectal cancer but not breast cancer. J Clinl Oncol 35: e23024, 2017.

33. Pozzoli G, Marei HE, Althani A, Boninsegna A, Casalbore P, Marlier LN, Lanzilli G, Zonfrillo M, Petrucci G, Rocca B, et al: Aspirin inhibits cancer stem cells properties and growth of glioblastoma multiforme through Rb1 pathway modulation. J Cell Physiol: Jan 30,2019 (Epub ahead of print). doi: 10.1002/jcp.28194.

34. Hull MA, Sprange K, Hepburn T, Tan W, Shafayat A, Rees CJ, Clifford G, Logan RF, Loadman PM, Williams EA, et al: Eicosapentaenoic acid and aspirin, alone and in combination, for the prevention of colorectal adenomas (seAFOod Polyp Prevention trial): A multicentre, randomised, double-blind, placebocontrolled, 2x2 factorial trial. Lancet 392: 2583-2594, 2018. 